\title{
Computer-based patient education is non-inferior to nurse counselling prior to colonoscopy: a multicenter randomized controlled trial
}

\author{
Authors \\ Joost P. H. Drenth ${ }^{1}$ \\ Institutions \\ 1 Department of Gastroenterology and Hepatology, \\ Radboud University Nijmegen Medical Centre, \\ Nijmegen, The Netherlands \\ 2 Department of Gastroenterology and Hepatology, \\ Northwest Hospital Group, Alkmaar, The Netherlands \\ 3 Department of Gastroenterology and Hepatology, \\ Jeroen Bosch Hospital, Den Bosch, The Netherlands \\ 4 Department of Gastroenterology and Hepatology, \\ Bernhoven Hospital, Uden, The Netherlands
}

Govert Veldhuijzen ${ }^{1}$, Michael Klemt-Kropp², Jochim S. Terhaar sive Droste ${ }^{3}$, Bas van Balkom4, Aura A. J. van Esch¹,

submitted 25.11 .2019

accepted after revision 23.7.2020

published online 23.7.2020

Bibliography

Endoscopy 2021; 53: 254-263

DOI 10.1055/a-1225-8708

ISSN 0013-726X

(C) 2020. Thieme. All rights reserved.

Georg Thieme Verlag KG, Rüdigerstraße 14,

70469 Stuttgart, Germany

$\circledast$ Appendix 1-3, Tables 1s-5s

Supplementary material is available under

https://doi.org/10.1055/a-1225-8708

Corresponding author

Govert Veldhuijzen, MD, Department of Gastroenterology and Hepatology, Radboud University Nijmegen Medical Centre, PO Box 9101, 6500 HB Nijmegen, The Netherlands Fax: +31-24-3540103

govert.veldhuijzen@radboudumc.nl

\section{ABSTRACT}

Background Optimal patient education prior to colonoscopy improves adherence to instructions for bowel preparation and leads to cleaner colons. We developed computerbased education (CBE) supported by video and $3 \mathrm{D}$ animations. We hypothesized that $\mathrm{CBE}$ could replace nurse counselling without loss of bowel preparation quality during colonoscopy.

Methods We conducted a prospective, multicenter, endoscopist-blinded, non-inferiority randomized controlled trial. The primary outcome was adequate bowel preparation, evaluated using the Boston Bowel Preparation Scale (BBPS). Secondary outcome measures were: sickness absence for outpatient clinic visits; patient anxiety/satisfaction scores; and information recall. We included patients in four endoscopy units (rural, urban, and tertiary).

Results We screened 1035 eligible patients and randomized 845. After evaluation, 684 were included in the intention-to-treat (ITT) group. Subsequently, 497 patients were included in the per-protocol analysis, 217 in the nurse counselling and 280 in the CBE group. Baseline characteristics were similarly distributed among the groups. On perprotocol analysis, adequate bowel cleansing was achieved in $93.2 \%(261 / 280)$ of CBE patients, which was non-inferior to nurse-counselled patients (94.0\%; 204/217), with a difference of $-0.8 \%$ (95\% confidence interval $[\mathrm{Cl}]-5.1 \%$ to $3.5 \%$ ). Non-inferiority was confirmed in the ITT population. Sickness absence was significantly more frequent in nurse-counselled patients $(28.0 \%$ vs. $4.8 \%)$. In CBE patients, $21.5 \%$ needed additional information, with $3.0 \%$ needing an extra outpatient visit.

Conclusion CBE is non-inferior to nurse counselling in terms of bowel preparation during colonoscopy, with lower patient sickness leave. CBE may serve as an efficient educational tool to inform patients before colonoscopy in routine clinical practice.

\section{Introduction}

Colonoscopy is the gold standard to detect and remove precancerous colonic lesions, such as adenomas. Colonoscopy, performed under proper conditions, reduces cancer morbidity and mortality [1]. Optimal bowel preparation is a key prerequi- site to achieve a high adenoma detection rate. Inappropriately cleaned colons result in suboptimal detection of relevant lesions and lead to repeated colonoscopies and shorter surveillance intervals [2,3]. 
There are a number of patient-related factors associated with poorly prepared colons, such as acceptance of the volume of bowel preparation, inability to follow the instructions, reduced awareness of health behavior, and health illiteracy [4, 5]. Several strategies have been used to improve bowel preparation through optimizing patient education. This can be achieved by the use of simple instruction tools [6]. Efforts that involve direct patient contact, such as patient navigators or nurses with face-to-face counselling are the most effective [7]. This is also paramount for increasing adherence to colonoscopy screening programs [8].

Face-to-face patient counselling is resource-rich and timeconsuming. The high demand for colonoscopy services as a result of colorectal screening programs has led to a surge in interest for more efficient strategies with less personnel, while maintaining quality levels.

There is evidence to suggest that e-health interventions are effective in improving information transfer to patients [9]. Internet-based education offers a number of advantages: it visualizes information in a comprehensible format; it is consistent and accessible at any desired moment; and it provides the option to remind patients in a timely fashion [6].

We have developed a web-based platform consisting of $3 \mathrm{D}$ animations, video, and voiceover text to inform patients about the colonoscopy procedure and the preparations needed [10]. This program mimics the patient journey from pre-colonoscopy consultation in the outpatient clinic to discharge after the procedure. A single center observational study compared this platform to nurse counselling, with patients who followed this program having adequate bowel preparation [11]. Subsequent efforts helped to evolve this program into an interactive computer-based education (CBE) tool. The main improvement consists of the addition of two-way communication to make homebased use feasible, with substitution of all of the elements of nurse counselling [12].

The effects of home-based CBE performance in terms of the quality of bowel preparation or number of repeated procedures because of inadequately prepared colons are unknown. We hypothesized that CBE as a modality for patient education is equally effective as nurse counselling for optimal bowel preparation. We report here on our multicenter randomized controlled trial with a head-to-head comparison of traditional nurse counselling versus CBE.

\section{Methods}

\section{Study design and patients}

We performed a multicenter, prospective, endoscopist-blinded, randomized controlled trial. Patients were recruited from the gastroenterology departments at four hospitals in the Netherlands, including one academic, two urban, and one rural-based hospital. Patients were recruited and underwent their colonoscopy between September 2015 and December 2017.

We included adults referred for complete colonoscopy requiring bowel preparation who could provide informed consent. The exclusion criteria were: patients unwilling to participate; no internet access or relative with internet access; Dutch illiteracy; audiovisual or mental disabilities. An extensive version of the trial protocol was published previously [10].

Patients were involved in the development of the CBE, using focus groups for content feedback. The study group promoted the $\mathrm{CBE}$ to the wider public, being nominated in several jury and public award contests. The CBE won the public vote for the yearly award issued by the Dutch low literate patient society in 2015.

\section{Ethics}

The study was performed according to the principles of the 1975 Declaration of Helsinki and the CONSORT guidelines for reporting of results were followed. This study was approved by the Institutional Review Board (Medical Research Ethics Committee of the region Arnhem-Nijmegen, number 2015-1765). The trial is registered under the Dutch Trial Registry, NTR 5475. No commercial support was provided for the trial.

\section{Randomization}

Patients referred for colonoscopy were contacted in person or by a telephone call to judge their willingness to participate. We employed a structured script to explain the goals of the trial. The reasons for a patient's unwillingness to participate were recorded. After the patient had given consent to participate, the patient identification number and their email address were entered on a secure online tool (Appendix 1s, see onlineonly Supplementary Material). This tool randomized patients in a 1:1 ratio per trial site and subsequently informed them by automated email of the type of education they would receive, being either nurse counselling or CBE. All participating patients provided written informed consent.

\section{Study procedure}

After enrolment in the trial, patients were either invited for a nurse-counselling session or the CBE. The intervention group received a unique link via email (known in cybersecurity terms as a hash) that provided access to the web-based platform [11]. In addition to the original software, patients had to complete an online questionnaire on medication use and their medical history. This form was returned via the secure tool to the endoscopy unit. An automated evaluation tool screened for potential risk factors for undergoing colonoscopy. If no red flags (e. g. use of anticoagulant or antidiabetic drugs, or history of severe cardiopulmonary condition) were noted, the patient was directly invited for colonoscopy. In other cases, patients were contacted by telephone or scheduled for an additional outpatient visit. This was also recorded.

Each participating hospital's CBE had a tailored video and site-specific instructions. The application outlook used video scripts and $3 D$ animations that were the same in all four sites ( Fig.1). The CBE customized to the academic trial site is openly accessible via https://trials.medify.eu/cbe-colonoscopy.

The control group was invited by mail to our outpatient clinic for a routine nurse-counselling visit. The nurse explained the procedure in full, acquired the relevant information on sedation pre-assessment, and handed out a short written leaflet on purgative use [13]. 


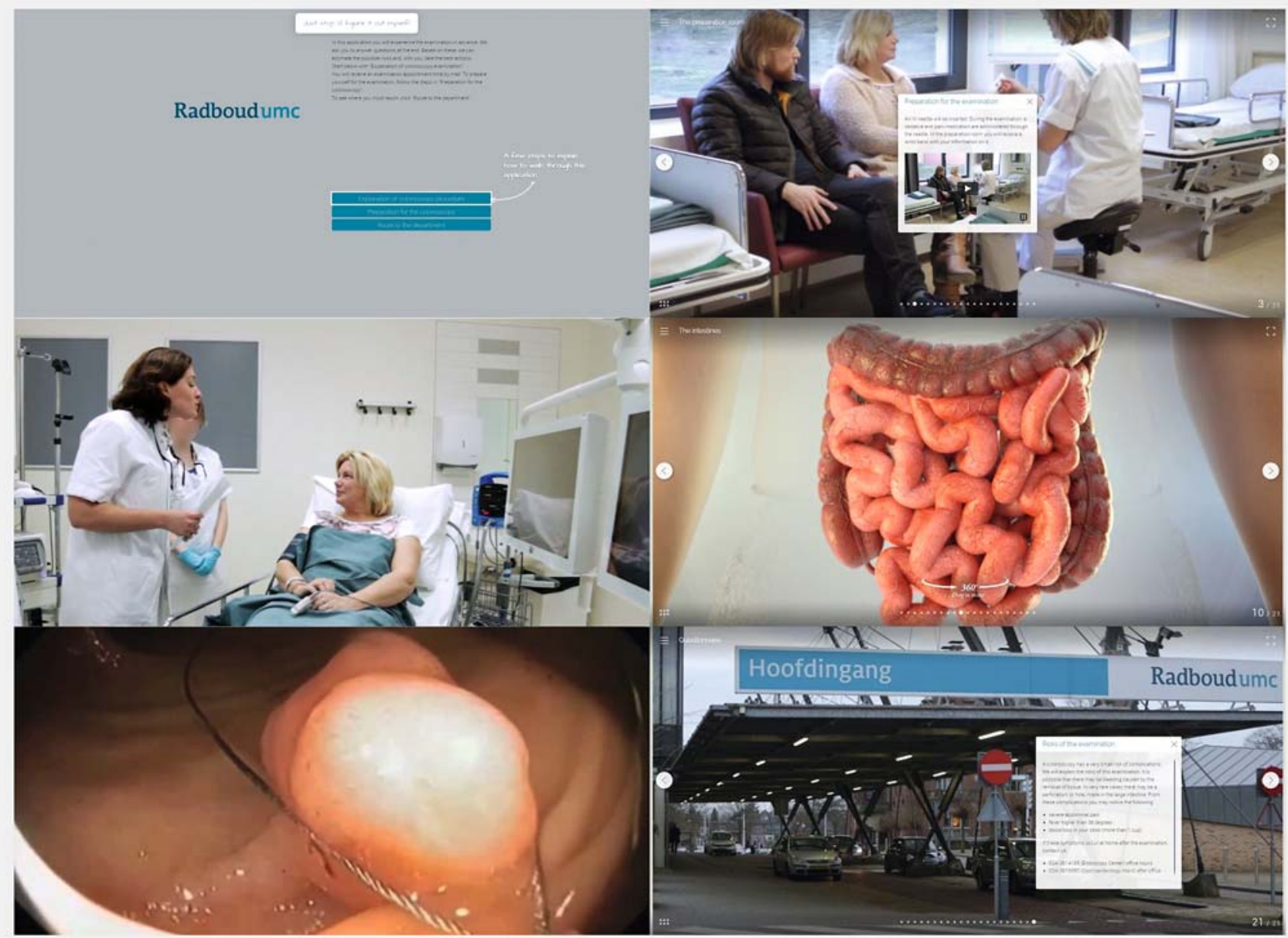

- Fig. 1 An overview of screenshots taken from the computer-based education that was used in this trial before colonoscopy, illustrating all the steps in the patients' journey (the individuals depicted are actors).

After completing either the CBE or nurse counselling, patients were scheduled for colonoscopy.

\section{Study design}

We used patient reported study questionnaires at several time points ( $\bullet$ Fig. 2 ). At the first time point (T1), baseline demographic characteristics, previous experience with colonoscopy, patient satisfaction, and validated questionnaires for e-health literacy and patient productivity were recorded $[14,15]$. After receiving the patient education (either nurse counselling or $\mathrm{CBE}$ ), the level of trait and state anxiety were measured [16].

On the day of colonoscopy, we collected patient information prior to colonoscopy (T2). Data on laxative use, the information recall test, and patient state anxiety were collected. Here, we also noted information on sickness absence leave [15]. In the CBE group, the need for additional contact events was scored.

The quality of the bowel preparation during colonoscopy was assessed by the Boston Bowel Preparation Scale (BBPS) [17]. Colonoscopy-specific data (indication, type of sedation and analgesic, ASA classification) were collected. Finally, prior to discharge (T3) patient satisfaction measures were recorded [18].

\section{Endoscopy}

The attending endoscopists were blinded to the type of education patients had received. All were familiarized or updated with the use of the BBPS before onset of the trial. The trial sites used either polyethylene glycol or sodium picosulfate-based standard split dose regimes for bowel preparation [19].

\section{Outcomes}

The primary outcome of the trial was quality of bowel preparation, as assessed with the BBPS. We recorded the need for repeat examinations owing to inadequate BBPS scores.

The secondary outcome measures were the patient-related outcome measures, including sickness absence leave, anxiety levels after instruction and prior to colonoscopy, satisfaction, and information recall.

\section{Questionnaires}

At baseline (T1), we included a validated questionnaire on health literacy (Dutch validated Health Literacy Scale) [14]. Anxiety levels were assessed at T1 using the State-Trait Anxiety Inventory (STAI). This commonly used 20-item self-report instru- 


\section{Adults referred for colonoscopy: assessment for eligibility and exclusion criteria}

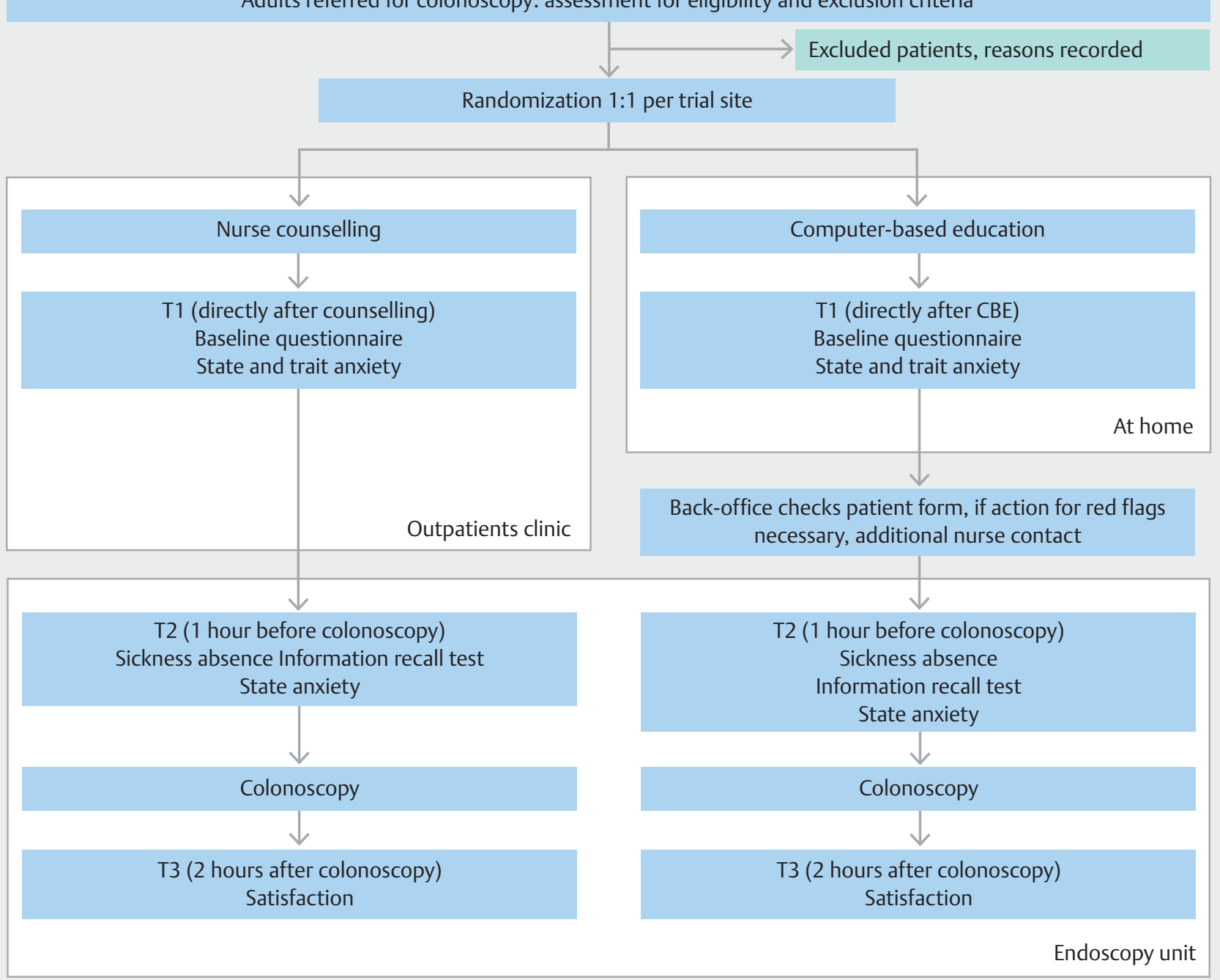

Fig. 2 Flowchart showing the assessments performed at the various time points.

ment provides scores ranging from 20 (absence of anxiety) to 80 (high anxiety) and combines both state (dynamic, at one particular moment) and trait (static, based on character) anxiety levels [16]. Patients reported sickness absence leave at T2 using the adapted iMTA Productivity Cost Questionnaire to evaluate the macroeconomic effect (Appendix 2s) [15]. Information recall was tested at $\mathrm{T} 2$ using the same 10 -item information recall test used in our prior pilot study [11].

For patient satisfaction, two measures were recorded at T3. First, the patients were asked about their willingness to return to the endoscopy unit. This is commonly used in the context of patient satisfaction in endoscopy [20]. Next, the Net Promoter Score (NPS) was used with the question "Would you recommend this endoscopy unit to your peers?" Patients' scores range from 1 (not at all likely) to 10 (extremely likely). The NPS is calculated as “\% promoters" (scores 9-10) - "\% Detractors" (scores 1-6) [18].

\section{Statistical analysis}

Data was analyzed on an intention-to-treat (ITT) and per-protocol basis. Presentation of the data included the mean, median, and standard deviations (SD) for quantitative data. The point estimates are presented with $95 \%$ confidence intervals $(\mathrm{Cl})$. For categorical data, counts and frequencies were used.

We used the relative risk of an inadequately prepared colon to compare the two groups. In comparable bowel preparation studies, a $90 \%$ success rate (for an adequately prepared colon) is commonly used, with a $10 \%$ non-inferiority margin as the maximum clinically acceptable difference $[19,21]$. The non-inferiority power calculation resulted in 180 patients per group, 360 patients in total. With a margin of $\pm 60 \%$ attrition of patients before completing the protocol, based on earlier research, the target number of patients to approach was set at 1000 to acquire an adequate per-protocol sample.

Comparisons between groups were assessed using bivariate analyses. In addition to the non-inferiority analyses in the ITT 


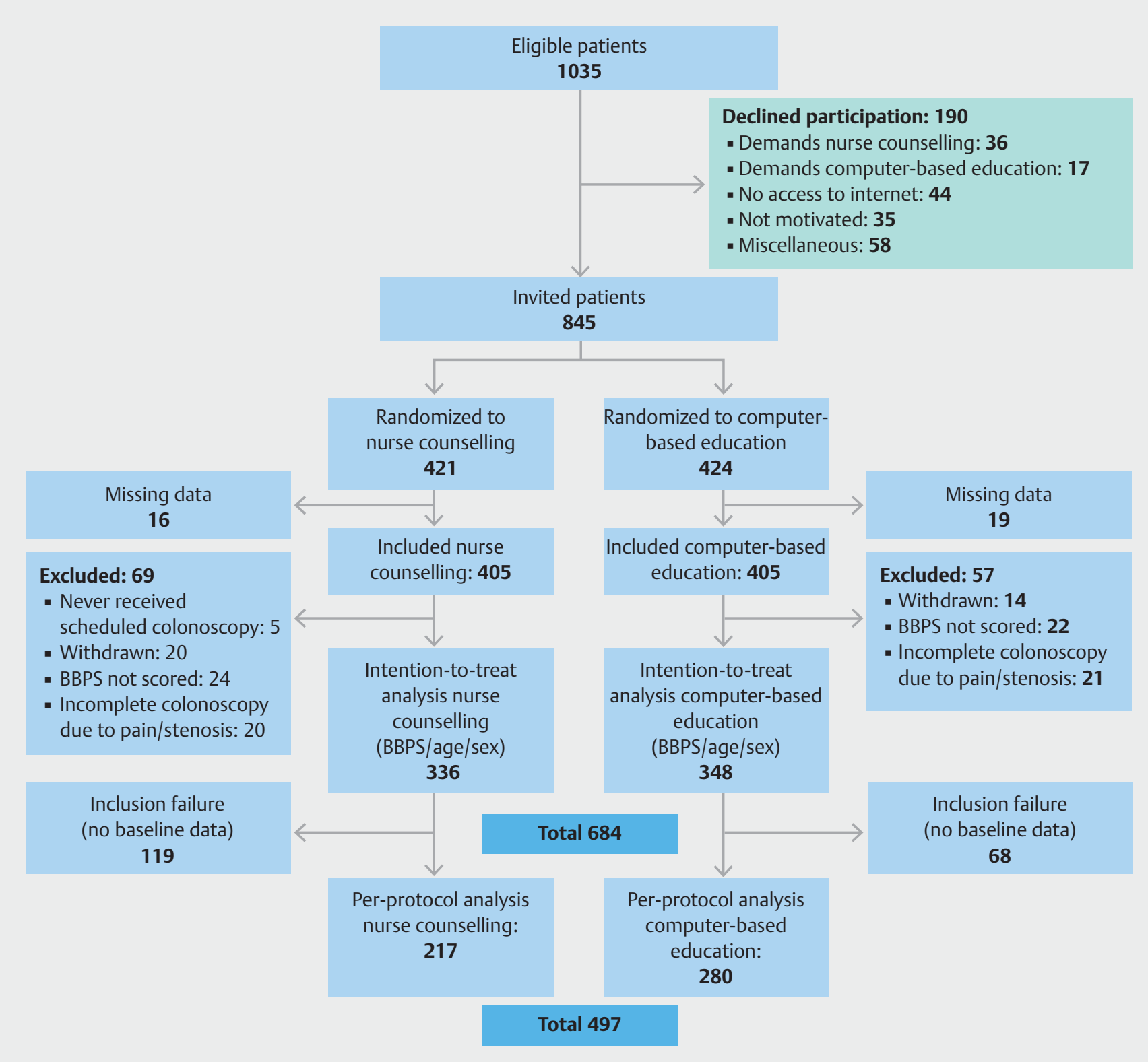

Fig.3 Flowchart of patient participation through the trial.

and per-protocol population, superiority analyses (chi-squares, $t$ test, and ANCOVA) were conducted to investigate effects on the secondary outcome measures. Possible differences between the groups concerning secondary outcomes were assessed using two-sided testing. $P$ values $<0.05$ were considered statistically significant for the secondary outcomes and no correction for multiple testing was performed as these analyses were considered exploratory.

\section{Results}

From September 2015 to December 2017, a total of 1035 patients were assessed for eligibility. Of those, 190 patients declined to participate for the reasons shown in $>$ Fig. 3. A total of 845 patients underwent randomization in four hospitals. After randomization, 161 patients were excluded from further analysis for the following five reasons: missing data $(n=35)$, premature withdrawal from the trial $(n=34)$, not receiving a scheduled colonoscopy $(n=5)$, an incomplete colonoscopy due to pain / stenosis ( $n=41)$, or absence of BBPS score $(n=46)$. This resulted in 684 patients who were included in the ITT analysis population. In these patients, the age and sex were not significantly different between the groups.

A total of 497 patients were entered for the per-protocol analysis. A total of 217 patients received nurse counselling, while 280 patients were assigned to CBE ( $\triangleright$ Fig. 3). All patients included in the analysis had $100 \%$ adherence to nurse counselling and to the complete CBE. Of these 497 patients, $100 \%$ completed 
- Table 1 Baseline characteristics of the 497 patients included in the per-protocol analysis.

\begin{tabular}{|c|c|c|c|}
\hline & $\begin{array}{l}\text { Nurse counselling } \\
(n=217)\end{array}$ & $\begin{array}{l}\text { Computer-based education } \\
(n=280)\end{array}$ & $\begin{array}{l}\text { P value } \\
\text { Nurse versus computer- } \\
\text { based education }{ }^{1}\end{array}$ \\
\hline \multicolumn{3}{|l|}{ Sex, $\mathrm{n}(\%)$} & \multirow[t]{3}{*}{0.54} \\
\hline - Male & $106(48.8)$ & $129(46.1)$ & \\
\hline - Female & $111(51.2)$ & $151(53.9)$ & \\
\hline Age, mean (SD), years & 56 years $(14.5)$ & 56 years $(14.5)$ & $0.80^{2}$ \\
\hline \multicolumn{3}{|l|}{ Ethnicity, n (\%) } & \multirow[t]{3}{*}{0.76} \\
\hline - Native Dutch & $194(89.4)$ & $242(86.4)$ & \\
\hline - Other & $22(10.1)$ & $30(10.7)$ & \\
\hline \multicolumn{3}{|l|}{ Educational level, n (\%) ${ }^{3}$} & \multirow[t]{4}{*}{0.98} \\
\hline - Low & $27(12.4)$ & $41(14.6)$ & \\
\hline - Middle & $121(55.8)$ & $154(55.0)$ & \\
\hline - High & $69(31.8)$ & $85(30.4)$ & \\
\hline Prior experience with colonoscopy, n (\%) & $101(46.8)$ & $140(51.5)$ & 0.30 \\
\hline \multicolumn{4}{|c|}{$\begin{array}{l}{ }^{1} \text { Chi-squared test, unless otherwise specified. } \\
{ }^{2} t \text { test. } \\
{ }^{3} \text { Highest completed educational level was split into three levels: "low," no education through to lower secondary education; "middle," upper secondary and middle } \\
\text { vocational education; "high," higher vocational and tertiary education. }\end{array}$} \\
\hline
\end{tabular}

the baseline study forms at $\mathrm{T} 1$, with lower response rates on pre-colonoscopy (T2) forms (55.6\%) and post-colonoscopy (T3) forms (47.3\%). The baseline characteristics of the patients were not significantly different with respect to age, sex, educational level, or ethnicity. Prior experience with colonoscopy was comparable between the two groups, with $46.8 \%$ in the nursecounselled group and $51.5 \%$ in the CBE group ( $>$ Table 1 ).

\section{Primary endpoint}

BBPS scores were collected for all 684 patients. In the ITT and per-protocol population, mean BBPS scores in both groups exceeded the threshold of 6 that is considered adequate. In the per-protocol population, the mean BBPS in the nurse-counselling group was 8.00 ( $95 \% \mathrm{Cl} 7.78$ to 8.21$)$, which was comparable to that of the CBE group at $7.81(95 \% \mathrm{Cl} 7.62$ to $8.00 ; P=0.21)$.

We subsequently calculated the risk of obtaining an adequate BBPS (>6). In the ITT population, among the CBE group, $93.4 \%$ of patients reached an adequate BBPS score, compared with $95.8 \%$ assigned to the nurse-counselling group. The $95 \% \mathrm{Cl}$ of the relative risk difference $(-2.4 \%)$ was $-5.8 \%$ to $0.9 \%$, which was within the prespecified non-inferiority margin of $10 \%$.

In the per-protocol population, among the CBE group, 93.2 $\%$ of patients reached an adequate BBPS score, compared with $94.0 \%$ assigned to the nurse-counselling group. The $95 \% \mathrm{Cl}$ of the relative risk difference $(-0.8 \%)$ was $-5.1 \%$ to $3.5 \%$, which was also within the prespecified non-inferiority margin of $10 \%$. Therefore, our findings show that CBE is non-inferior to nurse counselling in both the ITT and per-protocol population (i.e. that the null hypothesis was rejected).
The difference of $-2.4 \%(95 \% \mathrm{Cl}-5.8 \%$ to $0.9 \%)$ and $-0.8 \%$ ( $95 \% \mathrm{Cl}-5.1 \%$ to $3.5 \%)$ in formal testing for superiority showed no statistical difference between the groups in either the ITT or per-protocol population. The numbers of repeat colonoscopies owing to inadequate bowel preparation were not significantly different between the groups in either the ITT or perprotocol population, being $4(0.6 \%)$ for nurse counselling versus $7(1.0 \%)$ for CBE in the ITT group, and $3(0.6 \%)$ for nurse counselling versus $6(1.2 \%)$ for CBE in the per-protocol group ( $>$ Table 2).

Subsegmental BBPS scores in the right, transverse, and left colon were equally distributed amongst the groups (Table 1s). For excellent BBPS scores (8 or higher), no significant differences were observed between the groups (73.3\% for nurse counselling vs. $69.3 \%$ for $C B E ; P=0.33$ ) (Table 2 s).

\section{Secondary end points}

Sickness absence leave

Sickness absence leave was significantly lower in the CBE group at $28.0 \%$ in the nurse-counselling group and $4.8 \%$ in the CBE group $(P<0.001)$ ( $>$ Table 3$)$.

\section{Anxiety}

Anxiety scores were completed by 235 patients in total, 111 in the nurse counselling and 124 in the CBE group at baseline and sequentially before colonoscopy using the STAI. The baseline trait and state anxiety scores were equally distributed in the two groups: 53.4 (SD 5.3) in the nurse counselling versus 53.2 (SD 4.8) in the CBE group. This was also the case in state anxiety 
- Table 2 Bowel preparation assessed by the Boston Bowel Preparation Scale (BBPS) during colonoscopy for patients included in the per-protocol analysis.

\begin{tabular}{|c|c|c|c|}
\hline & $\begin{array}{l}\text { Nurse } \\
\text { counselling }\end{array}$ & $\begin{array}{l}\text { Computer-based } \\
\text { education) }\end{array}$ & $\begin{array}{l}P \text { value } \\
\text { Nurse versus computer-based edu- } \\
\text { cation }^{1}\end{array}$ \\
\hline Rate of adequate bowel preparation (BBPS $\geq 6$ ), $n(\%)$ & $204(94.0)$ & $261(93.2)$ & $\begin{array}{l}0.72 \text { for superiority } \\
\text { Non-inferiority: } \delta-0.8 \%(95 \% \mathrm{Cl}-5.1 \\
\text { to } 3.5)^{2}\end{array}$ \\
\hline BBPS score, mean $(95 \% \mathrm{Cl})$ & $\begin{array}{l}8.00 \\
(7.78 \text { to } 8.21)\end{array}$ & $\begin{array}{l}7.81 \\
(7.62 \text { to } 8.00)\end{array}$ & $0.21^{3}$ \\
\hline $\begin{array}{l}\text { Decision to repeat colonoscopy owing to inadequate bowel } \\
\text { preparation, } \mathrm{n}(\%)\end{array}$ & $3(1.4)$ & $6(2.1)$ & 0.53 \\
\hline $\begin{array}{l}\mathrm{Cl} \text {, confidence interval. } \\
{ }^{1} \text { Chi-squared test, unless otherwise specified. } \\
{ }^{2} \text { Within margin. } \\
{ }^{3} t \text { test. }\end{array}$ & & & \\
\hline
\end{tabular}

- Table 3 Comparison of the secondary outcomes (need for sickness leave, anxiety, satisfaction, and information recall).

\begin{tabular}{|c|c|c|c|}
\hline & $\begin{array}{l}\text { Nurse } \\
\text { counselling }\end{array}$ & $\begin{array}{l}\text { Computer-based } \\
\text { education }\end{array}$ & $\begin{array}{l}P \text { value } \\
\text { Nurse versus computer- } \\
\text { based education }{ }^{1}\end{array}$ \\
\hline $\begin{array}{l}\text { Need for sickness absence leave, n (\%) } \\
\text { [number of respondents, (\%)] }\end{array}$ & $\begin{array}{l}35(28.0) \\
{[125(57.3)]}\end{array}$ & $\begin{array}{l}7(4.8) \\
{[145(51.8)]}\end{array}$ & $0.001^{2}$ \\
\hline \multicolumn{4}{|l|}{$\begin{array}{l}\text { Anxiety (STAI) score }{ }^{3} \text {, mean (SD) } \\
\text { [number of respondents (\%)] }\end{array}$} \\
\hline Trait anxiety & $\begin{array}{l}53.4(5.3) \\
{[202(93.1)]}\end{array}$ & $\begin{array}{l}53.2(4.8) \\
{[212(75.7)]}\end{array}$ & 0.52 \\
\hline State anxiety after patient education & $\begin{array}{l}55.3(5.5) \\
{[203(93.5)]}\end{array}$ & $\begin{array}{l}54.4(5.8) \\
{[218(77.9)]}\end{array}$ & 0.10 \\
\hline State anxiety pre-colonoscopy & $\begin{array}{l}58.2(5.7) \\
{[118(54.4)]}\end{array}$ & $\begin{array}{l}578(5.3) \\
{[144(51.4)]}\end{array}$ & 0.65 \\
\hline Rise in state anxiety after education and prior to colonoscopy & $\begin{array}{l}3.1(7.0) \\
{[111(51.2)]}\end{array}$ & $\begin{array}{l}2.8(7.9) \\
{[124(44.3)]}\end{array}$ & $0.44^{4}$ \\
\hline \multicolumn{4}{|l|}{$\begin{array}{l}\text { Patient satisfaction } \\
\text { [number of respondents (\%)] }\end{array}$} \\
\hline Net promoter score ${ }^{5}$ & $\begin{array}{l}+40.9 \% \\
{[110(50.7)]}\end{array}$ & $\begin{array}{l}+46.3 \% \\
{[121(43.2)]}\end{array}$ & 0.45 \\
\hline Willingness to return (scale $1-10$ ), mean (SD) & $\begin{array}{l}8.13(1.35) \\
{[110(50.7)]}\end{array}$ & $\begin{array}{l}8.51(1.70) \\
{[121(43.2)]}\end{array}$ & 0.06 \\
\hline Information recall test ${ }^{6}$, mean (SD) & $\begin{array}{l}7.18(1.17) \\
{[125(57.6)]}\end{array}$ & $\begin{array}{l}7.24(1.06) \\
{[144(51.4)]}\end{array}$ & 0.70 \\
\hline \multicolumn{4}{|l|}{$\begin{array}{l}\text { STAI, State-Trait Anxiety Inventory; SD, standard deviation. } \\
{ }^{1} \text { Independent sample } t \text { test unless otherwise specified. } \\
{ }^{2} \text { Chi-squared test. } \\
{ }^{3} \text { Range from } 20 \text { (no anxiety) to } 80 \text { (high anxiety). } \\
{ }^{4} \text { ANCOVA. } \\
5 \text { \%promoters minus \%detractors. } \\
{ }^{6} 10 \text { basic-item test score before endoscopy (score range } 1-10 \text { ). }\end{array}$} \\
\hline
\end{tabular}

prior to colonoscopy. Comparing both groups, we noted an expected rise in anxiety scores between the moment of education and just before colonoscopy. The small difference in the rise in scores between the groups, indicating a possible benefit of either modality, was not significant: 3.1 (SD 7.0) versus 2.8 (SD 7.9); $P=0.44$ ( Table 3 ). 


\section{Satisfaction}

Patient satisfaction scores with the education before colonoscopy (defined as willingness to return) were high but not statistically different between the groups. The nurse-counselling group scored a mean of 8.13 (SD 1.35) out of 10, whereas the CBE group scored 8.55 (SD 1.30); $P=0.059$. Second, the NETpromoter scores recorded were $+40.9 \%$ versus $+46.3 \%$, respectively, which is also not significant amongst groups $(P=$ 0.45) ( Table 3).

\section{Information recall}

Information recall was tested using a 10 -item questionnaire. There was no significant difference between the groups, at 7.18 (SD 1.17) in the nurse-counselling group, versus 7.24 (SD 1.06 ) in the CBE group ( $\triangleright$ Table 3 ).

\section{Endoscopy}

When asked whether they required additional information prior to the colonoscopy, among the CBE group, $78.5 \%$ of patients responded negatively and were directly scheduled for colonoscopy after CBE. In $21.5 \%$ of cases, there was an extra contact event: $18.5 \%$ by telephone call and $3.0 \%$ at the outpatient clinic.

A total of 70 endoscopists were involved in the trial.

\section{Discussion}

This multicenter randomized controlled trial evaluated CBE as an educational tool for patient counselling prior to colonoscopy. We found in our ITT analysis, as well as on per-protocol analysis, that $C B E$ is non inferior to nurse counselling in terms of bowel preparation. At the same time, CBE reduced the number of patient visits to the outpatient clinic by $79 \%$ compared with conventional nurse counselling. An added value of CBE is the lower proportion of patients who report taking sickness absence leave prior to endoscopy. CBE, with two-way communication in place, functions therefore as a time- and resource-effective nexus between patients and the endoscopy unit.

We also investigated the psychological parameters, such as stress or anxiety, that may accompany (preparation for) a colonoscopy and found that there were no differences in the trait (or "character") anxiety scores between the groups. Similarly, the state (or "moment") anxiety scores were comparable between the groups both after receiving education and just prior to colonoscopy. Finally, CBE showed high scores for patient satisfaction (Appendix 3s) and information recall at levels similar to those seen after nurse counselling.

There have been several comparable studies that have used various means of electronic communication. One study enriched patient communication by sending a series of 15 text messages to patients and found that this led to better colonoscopy preparation being achieved [22]. In addition, digital send instructions increase appointment adherence with less sameday cancellations [23]. Trials using smartphone apps have shown improved bowel preparation [24-26]. An important difference with these studies using text messages via SMS or a smartphone app is that our approach aimed to achieve patient engagement through the use of visual $3 \mathrm{D}$ animation as a teach- ing tool to provide better insight and actual visualization of the procedure. Also, web-based solutions like ours have the benefit over smartphone apps that they are ubiquitously available on all devices (e.g. desktop computer, tablet, or smartphone) without the need for users to download anything first. More importantly, the fact that our CBE platform may substitute nurse counselling, a common practice in several healthcare services, is a novel element and relevant to policy makers [27].

Previously, it has been hypothesized that there is a "ceiling effect" of $90 \%$ adequate bowel preparation score for educational interventions that aim to influence the scores in any endoscopy unit [28]. As a result, these interventions will be beneficial in underperforming units with scores well below the $85 \%$ benchmark that has been advised by the US Multi-Society Task Force on Colorectal Cancer Screening [29]. In several recent (non-Western) studies that demonstrated improved bowel cleanliness by smartphone intervention, baseline scores in the control group were often below this point $(73.6 \%-77.2 \%)$ $[25,30]$. By contrast, in our four trial units (already performing well above $90 \%$ adequate bowel preparation in controls), the ceiling effect might have prevented the detection of any meaningful superiority difference. We therefore adopted the non-inferiority design, which was novel to this type of research.

Initiatives in other fields using the same functionality of CBE have shown that it can reduce the number of outpatient visits. For example, use of CBE improves patient self-management in inflammatory bowel disease, diabetes, asthma, and chronic obstructive pulmonary disorder [31, 32].

We realize that $C B E$ is not suitable for every patient. Patients with low (e-health) literacy are less likely to benefit. In our trial, $3.0 \%$ of patients paid an extra visit to the hospital despite CBE. CBE should therefore be positioned as an adjunct to nurse counselling in vulnerable patient groups, as they might need alternative access to relevant healthcare information.

The implication of our finding is that CBE may save valuable time for nurses and free up resources. With the growing future need for colonoscopies, due to the national colorectal cancer screening program and subsequent surveillance colonoscopies, and the current problems in recruiting nursing staff in Dutch hospitals, this is very relevant $[33,34]$.

Our randomized clinical trial comes with strengths and limitations. In terms of strengths, our trial was conducted with a large real-life sample of patients. The non-inferiority hypothesis and power allowed robust statements on the efficacy of CBE. We tested this CBE in a real-world setting, with patients having a variety of indications (Table 3s), both with and without previous experience of colonoscopy. Also, we used three different types of endoscopy unit, using a variety of different practices (Tables $4 \mathbf{s}$ and $\mathbf{5 s}$ ), so the results are generalizable to daily practice. In the catchment area of our endoscopy centers, CBE can be used in up to $94 \%$ of patients undergoing colonoscopy [11].

On the other hand, our trial comes with limitations. There were a significant number of drop outs after randomization owing to inclusion failures; however, this did not result in an unequal distribution regarding baseline characteristics between the arms in either the ITT or per-protocol populations, limiting the risk of selection bias. Because of the use of patient reported 
questionnaires, we do not have $100 \%$ data collection at all time points, although the trial protocol called for that. While this did not affect our main outcome, it might have affected the assessment of secondary outcomes, such as anxiety and satisfaction. Satisfaction was measured several hours after administration of sedatives. Sedatives may cause a euphoric effect after administration and result in higher overall scores. However, the types of sedative used were distributed equally over the groups (data not shown), precluding bias.

We did not collect complete medical histories of our patients, including previous abdominal surgery, or risk factors for poor bowel preparation, such as diabetes mellitus, constipation, or the use of motility influencing drugs. We surmise that the effect of these risk factors on the bowel preparation efficacy in our trial is limited, in view of the small difference in BBPS scores. We did not collect data on adenoma detection rate (ADR) as this was outside the remit of this clinical trial. From the literature, the robust correlation between adequate BBPS and ADR suggests that BBPS is a good technical proxy parameter [35].

In conclusion, in this trial, we have established non-inferiority for CBE compared with nurse counselling prior to colonoscopy in terms of bowel preparation. This finding paves the way for further upscaling of CBE in endoscopy units to prepare their patients more effectively before colonoscopy. Preparing patients with CBE reduces the need for outpatient clinic capacity, leading to less absenteeism at work, high satisfaction scores, and good recollection of information.

\section{Acknowledgments}

The authors would like to acknowledge Dr. Wietske Kievit and Dr. Reinier Akkermans for their methodological support and comments on the manuscript.

Funding support for this study was provided by ZonMW (The Netherlands Organisation for Health Research and Development) through a grant in the program "Actieplan eHealth," number 430000016 . Medify (producer of the software) had no role in the funding of this trial, nor in the design and conduct of the study, or writing and submission of the manuscript.

An abstract of this study has been presented at: the Dutch Association for Gastroenterology, Spring conference 2019, in Veldhoven, the Netherlands; the European Society of Gastrointestinal Endoscopy conference 2019, in Prague, Czech Republic; and the Digestive Disease Week 2019, in San Diego, USA.

\section{Competing interests}

The authors declare that they have no conflict of interest.

Clinical trial

Trial Registration: Netherlands Trial Register | Registration number (trial ID): NTR5475 | Type of study: Prospective, multicenter, endoscopist blinded, non-inferiority randomized controlled study.
References

[1] Corley DA, Jensen CD, Marks AR et al. Adenoma detection rate and risk of colorectal cancer and death. NEJM 2014; 370: 1298-1306

[2] Rex DK, Imperiale TF, Latinovich DR et al. Impact of bowel preparation on efficiency and cost of colonoscopy. Am J Gastroenterol 2002; 97: 1696-1700

[3] Rosenfeld G, Krygier D, Enns RA et al. The impact of patient education on the quality of inpatient bowel preparation for colonoscopy. Can J Gastroenterol 2010; 24: 543-546

[4] Rex DK. Optimal bowel preparation-a practical guide for clinicians. Nat Rev Gastroenterol Hepatol 2014; 11: 419-425

[5] Waldmann E, Penz D, Majcher B et al. Impact of high-volume, intermediate-volume and low-volume bowel preparation on colonoscopy quality and patient satisfaction: An observational study. United European Gastroenterol J 2019; 7: 114-124

[6] Liu Z, Zhang MM, Li YY et al. Enhanced education for bowel preparation before colonoscopy: A state-of-the-art review. J Dig Dis 2017; 18 : 84-91

[7] Abuksis G, Mor M, Segal N et al. A patient education program is costeffective for preventing failure of endoscopic procedures in a gastroenterology department. Am J Gastroenterol 2001; 96: 1786-1790

[8] Stratmann $\mathrm{K}$, Bock H, Filmann $\mathrm{N}$ et al. Individual invitation letters lead to significant increase in attendance for screening colonoscopies: Results of a pilot study in Northern Hesse, Germany. United European Gastroenterol J 2018; 6: 1082-1088

[9] Suhling H, Rademacher J, Zinowsky I et al. Conventional vs. tablet computer-based patient education following lung transplantation-a randomized controlled trial. PLoS One 2014; 9: e90828

[10] Veldhuijzen G, van Esch AA, Klemt-Kropp M et al. E-Patient Counseling Trial (E-PACO): Computer based education versus nurse counseling for patients to prepare for colonoscopy. J Vis Exp 2019: doi: $10.3791 / 58798$

[11] Veldhuijzen G, Klemt-Kropp M, Noomen C et al. Computer-assisted instruction before colonoscopy is as effective as nurse counselling, a clinical pilot trial. Endosc Int Open 2017; 5: E792-E797

[12] Fox MP. A systematic review of the literature reporting on studies that examined the impact of interactive, computer-based patient education programs. Patient Educ Couns 2009; 77: 6-13

[13] Munsterman ID, Cleeren E, van der Ploeg T et al. 'Pico-Bello-Klean study': effectiveness and patient tolerability of bowel preparation agents sodium picosulphate-magnesium citrate and polyethylene glycol before colonoscopy. A single-blinded randomized trial. Eur J Gastroenterol Hepatol 2015; 27: 29-38

[14] van der Vaart R, Drossaert CH, Taal E et al. Validation of the Dutch functional, communicative and critical health literacy scales. Patient Educ Couns 2012; 89: 82-88

[15] Bouwmans C, Krol M, Severens $\mathrm{H}$ et al. The iMTA Productivity Cost Questionnaire: A standardized instrument for measuring and valuing health-related productivity losses. Value Health 2015; 18: 753-758

[16] Spielberger CD, Gorsuch R, Lushene RE, Vagg PR. Manual for the State-Trait Anxiety Inventory. Palo Alto, California, USA: Consulting Psychologists Press; 1983

[17] Mittal S. The Boston Bowel Preparation Scale: reliable not only for colonoscopy-oriented research but clinical practice also. Gastrointest Endosc 2010; 71: 221

[18] Krol MW, de Boer D, Delnoij DM et al. The Net Promoter Score - an asset to patient experience surveys? Health Expect 2015; 18: 30993109

[19] Schreiber S, Baumgart DC, Drenth JPH et al. Colon cleansing efficacy and safety with $1 \mathrm{~L}$ NER1006 versus sodium picosulfate with magnesium citrate: a randomized phase 3 trial. Endoscopy 2019; 51: 73-84 
[20] Loftus R, Nugent Z, Graff LA et al. Patient satisfaction with the endoscopy experience and willingness to return in a central Canadian health region. Can J Gastroenterol 2013; 27: 259-266

[21] DeMicco MP, Clayton LB, Pilot J et al. Novel $1 \mathrm{~L}$ polyethylene glycolbased bowel preparation NER1006 for overall and right-sided colon cleansing: a randomized controlled phase 3 trial versus trisulfate. Gastrointest Endosc 2018; 87: 677-687 e673

[22] Walter B, Klare P, Strehle K et al. Improving the quality and acceptance of colonoscopy preparation by reinforced patient education with short message service: results from a randomized, multicenter study (PERICLES-II). Gastrointest Endosc 2019; 89: 506-513 e504

[23] Richter JM, Ha JB, Marx M et al. A digital preprocedure instruction program for outpatient colonoscopy. Telemed J E Health 2020; 26: 468-476

[24] Lorenzo-Zuniga V, Moreno de Vega V, Marin I et al. Improving the quality of colonoscopy bowel preparation using a smart phone application: a randomized trial. Dig Endosc 2015; 27: 590-595

[25] Sharara Al, Chalhoub JM, Beydoun M et al. A customized mobile application in colonoscopy preparation: a randomized controlled trial. Clin Transl Gastroenterol 2017; 8: e211

[26] Desai M, Nutalapati V, Bansal A et al. Use of smartphone applications to improve quality of bowel preparation for colonoscopy: a systematic review and meta-analysis. Endosc Int Open 2019; 7: E216-E224

[27] Nelson MJ, Keswani RN. Inadequate colonoscopy preparation: Is it time to send out an SMS? Gastrointest Endosc 2019; 89: 514-517

[28] MacArthur KL, Leszczynski AM, Jacobson BC. Enhancing bowel preparation instructions: Is the bang worth the buck, or are we stuck with the muck? Gastrointest Endosc 2017; 85: 98-100
[29] Johnson DA, Barkun AN, Cohen LB et al. Optimizing adequacy of bowel cleansing for colonoscopy: recommendations from the US multi-society task force on colorectal cancer. Am J Gastroenterol 2014; 109: 1528

[30] Back SY, Kim HG, Ahn EM et al. Impact of patient audiovisual re-education via a smartphone on the quality of bowel preparation before colonoscopy: a single-blinded randomized study. Gastrointest Endosc 2018; 87: 789-799 e784

[31] de Jong MJ, van der Meulen-de Jong AE, Romberg-Camps MJ et al. Telemedicine for management of inflammatory bowel disease (mylBDcoach): a pragmatic, multicentre, randomised controlled trial. Lancet 2017; 390: 959-968

[32] Sangrar R, Docherty-Skippen SM, Beattie K. Blended face-to-face and online/computer-based education approaches in chronic disease selfmanagement: A critical interpretive synthesis. Patient Educ Couns 2019; 102: 1822-1832

[33] V\&VN. Rapport Arbeidsmarkt 2019 [In Dutch]. https://www.uwv.nl/ overuwv/Images/factsheet-arbeidsmarkt-zorg-maart2020.pdf Accessed: 9 September 2020

[34] Toes-Zoutendijk E, van Leerdam ME, Dekker E et al. Real-time monitoring of results during first year of Dutch colorectal cancer screening program and optimization by altering fecal immunochemical test cutoff levels. Gastroenterology 2017; 152: 767-775 e762

[35] Jain D, Singhal S. Factors affecting bowel preparation and adenoma detection: patient or the doctor. Gastrointest Endosc 2015; 82: 583 